\title{
DETERMINACIÓN DE LOS PARÁMETROS DE PROCESAMIENTO QUE MAXIMICEN EL RENDIMIENTO Y LA ESTABILIDAD DEL NÉCTAR DE UNGURAHUI (Oenocarpus bataua, C. Martius)
}

\author{
María Cecilia Fuentes Gómez ${ }^{1}$, Américo Guevara Pérez ${ }^{2 *}$
}

\begin{abstract}
RESUMEN
Se evaluó los parámetros de procesamiento que maximicen el rendimiento de la pulpa y la estabilidad del néctar de ungurahui, mediante las metodologías de Taguchi y Superficie de Respuesta. La materia prima utilizada procedió de la localidad de Yurimaguas, Región Loreto - Perú, y fue sometida a procesos de pesado, selección, clasificación, lavado, desinfectado, maduración, pulpeado, refinado, filtrado, estandarizado (para formular el néctar), pasteurizado, envasado, enfriado y almacenado. En la primera etapa se obtuvieron los parámetros de maduración y pulpeado $\left(\mathrm{T}^{\circ}: 51,99^{\circ} \mathrm{C}\right.$, tiempo: $1,74 \mathrm{~h}$, fruta recolectada: agua en la maduración (1:3), fruta madurada: agua en el pulpeado (1: 0,75$)$ ), que optimizaron el rendimiento $(37,33 \%)$ de pulpa de ungurahui. En la segunda etapa se determinó los parámetros de estandarizado (pulpa: agua $(1: 1,33)$, porcentaje de gomas: xantana $(0,02 \%)$; arábiga $(0,03 \%)$; carboximetil celulosa $(0,06 \%)$, y lecitina $(0,02 \%)$ ) que optimizaron la estabilidad (2,27 x10-8 ml (nivel de aro en la superficie de la botella)) del néctar de ungurahui. El producto terminado tuvo buena aceptabilidad de textura, sabor y color. Asimismo, los recuentos bajos de aerobios mesofilos viables $(10 \mathrm{UFC} / \mathrm{ml})$, levaduras $(<1 \mathrm{UFC} / \mathrm{ml})$ y mohos $(<1 \mathrm{UFC} / \mathrm{ml})$, aseguraron la calidad del producto obtenido.
\end{abstract}

Palabras clave: ungurahui, rendimiento, estabilidad, taguchi, superficie de respuesta.

\section{DETERMINATION OF PROCESSING PARAMETERS THAT MAXIMIZE THE PERFORMANCE AND STABILITY OF THE UNGURAHUI NECTAR (Oenocarpus bataua, C. Martius)}

\begin{abstract}
The processing parameters that maximize the pulp yield and the stability of the ungurahui nectar were evaluated by means of the Taguchi and Response Surface methodologies. The raw material used came from the town of Yurimaguas, Loreto Region - Peru, and was

\footnotetext{
${ }^{1}$ Facultad de Industrias Alimentarias, Universidad Nacional Agraria La Molina, Lima.

$2^{2 *}$ Profesor principal Facultad de Industrias Alimentarias, Universidad Nacional Agraria La Molina, Lima. aguevara@lamolina.edu.pe
} 
subjected to weighing, selection, classification, washing, disinfecting, maturing, pulping, refining, filtering, standardized (to formulate the nectar), pasteurized, packed, cooled and stored processes. In the first stage the parameters of maturation and pulping were obtained $\left(\mathrm{T}^{\circ}: 51,99^{\circ} \mathrm{C}\right.$, time: $1,74 \mathrm{~h}$, fruit harvested: water in maturation $(1: 3)$, ripened fruit: water in the pulped $(1: 0,75))$, which optimized the yield $(37,33 \%)$ of ungurahui pulp. In the second stage the standardized parameters were determined (pulp: water $(1: 1,33)$, percentage of gums: xanthan $(0,02 \%)$, arabica $(0,03 \%)$, carboxymethylcellulose $(0,06 \%)$ and lecithin $(0,02 \%))$ that optimized the stability $(2,27 \times 10-8 \mathrm{ml}$ (level of the ring on the surface of the bottle)) of the ungurahui nectar. The final product had a good acceptability of texture, taste and color. Likewise, low counts of viable mesophilic aerobes $(10 \mathrm{CFU} / \mathrm{ml})$, yeasts $(<1 \mathrm{CFU}$ $/ \mathrm{ml})$ and molds $(<1 \mathrm{CFU} / \mathrm{ml})$ ensured the quality of the product obtained.

Key words: ungurahui, performance, stability, taguchi and response surface.

\section{INTRODUCCIÓN}

Desde hace algunas décadas, el manejo forestal considera en el proceso de toma de decisiones tres factores: el económico, el social y el ecológico, orientando la cosecha de productos o la provisión de servicios ambientales de acuerdo con las capacidades de los ecosistemas ${ }^{1}$. Tiene entonces que ver con la sociedad y las personas, y la necesidad de que deban y puedan mantener y aumentar los servicios, beneficios económicos y la salud de los ecosistemas forestales para su desarrollo y mejor calidad de vida.

Las palmeras son uno de los grupos de plantas más diversas y económicamente valiosas en el mundo ${ }^{2}$. Una de ellas, identificada en el Perú como recurso prioritario para desarrollar alternativas económicas sostenibles locales y regionales, es el ungurahui (Oenocarpus bataua, C. Martius), por tener múltiples usos como alimento (bebidas, pulpa, palmito, aceite, alimento fresco y un medio para cultivar larvas de insectos (ricas en proteínas, para consumo humano), medicina, fibras, material de construcción, artesanías y objetos manuales ${ }^{3}$.

El ungurahui crece hasta los 950 msnm, de manera silvestre o cultivado en América Central y la Amazonia, sus frutos poseen muy buenas características físicas, organolépticas y químicas ( $21,30 \%$ de grasa con presencia de omegas 3, 6 y 9; 6,5\% de proteínas y $1,13 \%$ de cenizas), además de representar una alternativa viable como potencial materia prima para diferentes productos nutritivos industrializados, como lo es el néctar, cuya dificultad principal es la separación de la grasa, haciendo que su aprovechamiento sea básicamente en refrescos de consumo inmediato.

Por lo expuesto, esta investigación, fijó como objetivos, determinar los parámetros de procesamiento que maximicen el rendimiento y la estabilidad del néctar de ungurahui, así como la caracterización de la materia prima y producto obtenido. 


\section{PARTE EXPERIMENTAL}

La investigación se realizó en la planta piloto de la Facultad de Industrias Alimentarias, así como en los laboratorios acreditados de Fisicoquímica y Microbiología de La Molina Calidad Total Laboratorios; instalaciones pertenecientes a la Universidad Nacional Agraria La Molina.

\section{Materia prima e insumos}

Ungurahui, proveniente de la provincia de Yurimaguas - Región Loreto. Azúcar blanca refinada, agua potable tratada, carboximetil celulosa (CMC), goma arábiga, goma xantana, lecitina, ácido cítrico y sorbato de potasio.

\section{Equipos y materiales}

Colorímetro KONICA MINOLTA ${ }^{\circledR}$, potenciómetro digital WATERPROOF, refractómetro ECLIPSE, viscosímetro BROOKFIELD, equipo Semi-micro KJENDAHL®, equipo SOXLETH ${ }^{\circledR}$, AQUALAB®, estufa MERMMERT, pulpeadora refinadora REEVES, termómetro digital TRACEABLE Control Company, olla con chaqueta de vapor, balanza analítica digital TRAVELER TM, baño María GEL (Gesells Schaft Fur), materiales de vidrio/plástico, pinzas metálicas y placas de aluminio.

\section{Análisis fisicoquímico}

En los frutos de ungurahui, se determinó: humedad, proteína, grasa, fibra bruta, cenizas, sólidos solubles, $\mathrm{pH}$ y acidez, mediante los métodos oficiales de $\mathrm{AOAC}^{4}$ : 934.06, 920.152, 930.09, 930.10, 940.26, 932.12, 981.12, 942.15, respectivamente. También se determinó carbohidratos, por diferencia ${ }^{5}$. En el néctar de ungurahui, adicionalmente se determinó:

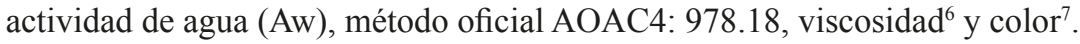

\section{Análisis microbiológico}

En el néctar de ungurahui, se determinó la numeración de bacterias aerobios mesófilos viables, mohos y levaduras, mediante los métodos recomendados por APHA/CMMEF ${ }^{8}$. Análisis sensorial del néctar de ungurahui

Se realizó con 100 jueces no entrenados, seleccionados previamente mediante una encuesta. A los jueces se les entregó un formato elaborado mediante una escala lineal estructurada de 10 $\mathrm{cm}$ de longitud, y se les pidió que marcaran en la escala preestablecida de diez puntos, desde me desagrada mucho $(0=$ escala inferior $)$ hasta me agrada mucho $(10=$ escala superior $)$.

\section{Metodología Experimental}

En la maduración-pulpeado y estandarizado, se optimizaron los factores que influyen significativamente $(\mathrm{p}<0,05)$ en el rendimiento de la pulpa y estabilidad del néctar de ungurahui, tal como se muestra en la figura 1. 
Optimización de los factores $(p<0,05)$ que influyen en el rendimiento de la pulpa de ungurahui

- En la maduración, ratio fruta: agua, temperatura y tiempo.

- En el pulpeado, ratio fruta: agua.

- Variable respuesta: rendimiento.

Determinación de los factores $(\mathbf{p}<0,05)$ que influyen en la estabilidad del néctar de ungurahui

- En el estandarizado, ratio pulpa: agua, concentración de: goma arábiga, goma xantana, CMC y lecitina.

- Variable respuesta: estabilidad (nivel de aro en la superficie de la botella).

Figura 1. Esquema experimental

\section{Evaluación estadística}

Para el análisis estadístico de los resultados se empleó las metodologías de Taguchi y Superficie de Respuesta, con un arreglo ortogonal L18 $\left(2 \times 3^{7-5}\right)$, para un DCA, usando el software estadístico Design Expert ${ }^{\circledR}$ Versión 7.0.0, con un nivel de significancia de $95 \%$ ( $\mathrm{p}<0,05)$, y criterios de "Más es Mejor" o "Nominal es Mejor”, según la variable respuesta considerada en cada etapa. Tal como se muestra en las tablas 1 y 2 , en cada etapa se obtuvieron 18 tratamientos, cuyos resultados fueron analizados por duplicado, mediante análisis de varianza (ANVA).

Tabla 1. Distribución de los factores y sus niveles en el arreglo ortogonal Taguchi L18 (2 x $3^{7-5}$ ), para la obtención de pulpa de ungurahui con máximo rendimiento

\begin{tabular}{|c|c|c|c|c|c|c|c|c|}
\hline \multirow{2}{*}{ Tratamientos } & \multicolumn{8}{|c|}{ Factores de Control } \\
\hline & $\mathrm{X1}$ & $\mathrm{X} 2$ & X3 & $\mathrm{X4}$ & $\mathrm{X5}$ & X6 & $\mathrm{X7}$ & X8 \\
\hline 1 & - & 40 & 1,50 & $1: 1$ & - & - & - & $1: 0,25$ \\
\hline 2 & - & 40 & 2,00 & $1: 2$ & - & - & - & $1: 0,50$ \\
\hline 3 & - & 40 & 2,50 & $1: 3$ & - & - & - & $1: 0,75$ \\
\hline 4 & - & 50 & 1,50 & $1: 1$ & - & - & - & $1: 0,75$ \\
\hline 5 & - & 50 & 2,00 & $1: 2$ & - & - & - & $1: 0,25$ \\
\hline 6 & - & 50 & 2,50 & $1: 3$ & - & - & - & $1: 0,50$ \\
\hline 7 & - & 60 & 1,50 & $1: 2$ & - & - & - & $1: 0,75$ \\
\hline 8 & - & 60 & 2,00 & $1: 3$ & - & - & - & $1: 0,25$ \\
\hline 9 & - & 60 & 2,50 & $1: 1$ & - & - & - & $1: 0,50$ \\
\hline 10 & - & 40 & 1,50 & $1: 3$ & - & - & - & $1: 0,25$ \\
\hline 11 & - & 40 & 2,00 & $1: 1$ & - & - & - & $1: 0,50$ \\
\hline 12 & - & 40 & 2,50 & $1: 2$ & - & - & - & $1: 0,75$ \\
\hline 13 & - & 50 & 1,50 & $1: 2$ & - & - & - & $1: 0,50$ \\
\hline 14 & - & 50 & 2,00 & $1: 3$ & - & - & - & $1: 0,75$ \\
\hline 15 & - & 50 & 2,50 & $1: 1$ & - & - & - & $1: 0,25$ \\
\hline 16 & - & 60 & 1,50 & $1: 3$ & - & - & - & $1: 0,50$ \\
\hline 17 & - & 60 & 2,00 & $1: 1$ & - & - & - & $1: 0,75$ \\
\hline 18 & - & 60 & 2,50 & $1: 2$ & - & - & - & $1: 0,25$ \\
\hline
\end{tabular}


Donde:

X2: $\quad$ Temperatura de maduración $\left({ }^{\circ} \mathrm{C}\right)$.

X3: $\quad$ Tiempo de maduración $(\mathrm{h})$.

X4: $\quad$ Ratio fruta: agua en la maduración (a: b).

X5: Interacción Temperatura - Tiempo de maduración.

X6: Interacción Ratio fruta: agua en la maduración - Tiempo de maduración.

X7: Interacción Temperatura de maduración - Ratio fruta: agua en la maduración.

X8: $\quad$ Ratio fruta: agua en el Pulpeado (a: c).

Tabla 2. Distribución de los factores y sus niveles en el arreglo ortogonal de Taguchi L18 $\left(2 \times 3^{7-5}\right)$, para la obtención de néctar de ungurahui con máxima estabilidad

\begin{tabular}{|c|c|c|c|c|c|c|c|c|}
\hline \multirow{2}{*}{ Tratamientos } & \multicolumn{8}{|c|}{ Factores de Control } \\
\hline & $\mathrm{X} 1$ & $\mathbf{X} 2$ & $\mathbf{X 3}$ & $\mathrm{X} 4$ & X5 & X6 & $\mathbf{X} 7$ & X8 \\
\hline 1 & - & $1: 1$ & 0,000 & 0,000 & 0,000 & 0,000 & - & - \\
\hline 2 & - & $1: 1$ & 0,025 & 0,050 & 0,025 & 0,300 & - & - \\
\hline 3 & - & $1: 1$ & 0,050 & 0,100 & 0,050 & 0,600 & - & - \\
\hline 4 & - & $1: 2$ & 0,000 & 0,000 & 0,025 & 0,300 & - & - \\
\hline 5 & - & $1: 2$ & 0,025 & 0,050 & 0,050 & 0,600 & - & - \\
\hline 6 & - & $1: 2$ & 0,050 & 0,100 & 0,000 & 0,000 & - & - \\
\hline 7 & - & $1: 3$ & 0,000 & 0,050 & 0,000 & 0,600 & - & - \\
\hline 8 & - & $1: 3$ & 0,025 & 0,100 & 0,025 & 0,000 & - & - \\
\hline 9 & - & $1: 3$ & 0,050 & 0,000 & 0,050 & 0,300 & - & - \\
\hline 10 & - & $1: 1$ & 0,000 & 0,100 & 0,050 & 0,300 & - & - \\
\hline 11 & - & $1: 1$ & 0,025 & 0,000 & 0,000 & 0,600 & - & - \\
\hline 12 & - & $1: 1$ & 0,050 & 0,050 & 0,025 & 0,000 & - & - \\
\hline 13 & - & $1: 2$ & 0,000 & 0,050 & 0,050 & 0,000 & - & - \\
\hline 14 & - & $1: 2$ & 0,025 & 0,100 & 0,000 & 0,300 & - & - \\
\hline 15 & - & $1: 2$ & 0,050 & 0,000 & 0,025 & 0,600 & - & - \\
\hline 16 & - & $1: 3$ & 0,000 & 0,100 & 0,025 & 0,600 & - & - \\
\hline 17 & - & $1: 3$ & 0,025 & 0,000 & 0,050 & 0,000 & - & - \\
\hline 18 & - & $1: 3$ & 0,050 & 0,050 & 0,000 & 0,300 & - & - \\
\hline
\end{tabular}

Donde:

X2: $\quad$ Ratio pulpa: agua (a: b)

X3: $\quad$ Goma Xantana (\%)

X4: $\quad$ CMC (\%)

X5: Goma Arábiga (\%)

X6: Lecitina (\%) 
En la figura 2 se muestra el flujo de operaciones seguido para elaborar néctar de ungurahui, se aprecia que posterior a la optimización, los ${ }^{\circ}$ Brix fueron regulados a 13 con azúcar blanca refinada y el pH a 3,7 con ácido cítrico.

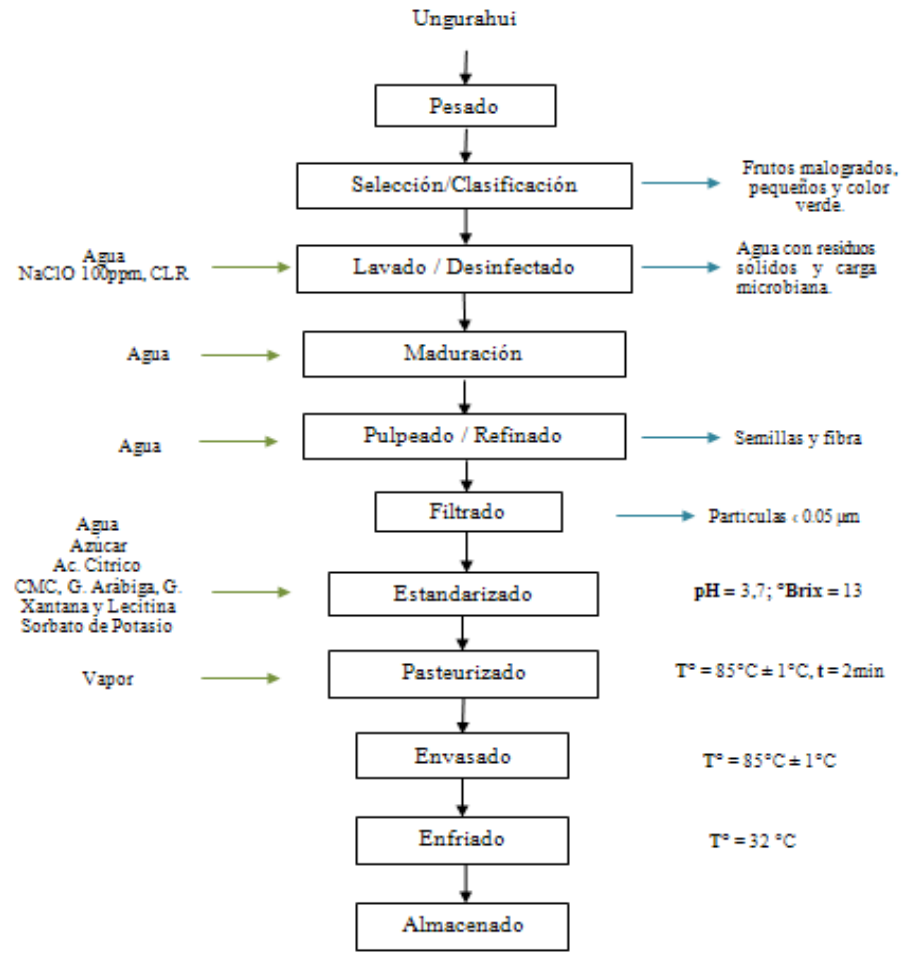

Figura 2. Flujo de operaciones para obtener néctar de ungurahui

\section{RESULTADOS Y DISCUSIÓN}

\section{Optimización de los factores $(\mathbf{p}<0,05)$ que influyen en el rendimiento de la pulpa de ungurahui.}

Mediante el análisis de varianza (ANVA), se determinó la significancia $(\mathrm{p}<0,0001)$ del modelo cuadrático descrito en la ecuación de la figura 3. La ecuación muestra el comportamiento del rendimiento de la pulpa de ungurahui ante cambios en los factores independientes considerados.

Rendimiento $(\mathrm{g} / \mathrm{kg})=-405,83+6,32 *$ Temperatura $+267,59 *$ Tiempo $-24,29 *$ Ratio Escaldado + 104, 34*Ratio Pulpeado 1,46*Temperatura*Tiempo -0,37*Temperatura*Ratio Escaldado -2,09*Temperatura*Ratio Pulpeado - 8,28*Tiempo*Ratio Escaldado -11,49*Tiempo*Ratio Pulpeado + 29,31*Ratio Escaldado*Ratio Pulpeado $-0,01^{*}$ Temperatura $^{2}-43,84 *$ Tiempo $^{2}+$ $11,49 *$ Ratio Escaldado ${ }^{2}-50 *$ Ratio Pulpeado ${ }^{2}$

Figura 3. Modelo cuadrático para optimizar el rendimiento de pulpa de ungurahui 
En la tabla 3 se muestra los factores independientes y sus niveles, considerados en el análisis de varianza (ANVA), para la optimización del rendimiento de la pulpa de ungurahui. Al respecto, el rendimiento de una fruta varía en función al cultivar y en muchas ocasiones, de su estado de desarrollo, índice de madurez y factores de procesamiento ${ }^{9}$.

Tabla 3. Optimización de la variable respuesta rendimiento

\begin{tabular}{|c|c|c|c|c|}
\hline \multicolumn{5}{|c|}{ Respuesta Óptima } \\
\hline \multicolumn{5}{|c|}{ Objetivo: Máximo Rendimiento de Pulpa de Ungurahui } \\
\hline \multicolumn{5}{|l|}{ Valor Óptimo = 43,25 } \\
\hline Factor & Menor & Medio & Mayor & Óptimo \\
\hline Temperatura $\left({ }^{\circ} \mathrm{C}\right)$ & 40 & 50 & 60 & 58 \\
\hline Tiempo (h) & 1,50 & 2,00 & 2,50 & 2,04 \\
\hline $\begin{array}{l}\text { Ratio: Maduración (a:b) } \\
\text { kg }\end{array}$ & $1: 1$ & $1: 2$ & $1: 3$ & $1: 1,03$ \\
\hline Ratio: Pulpeado (c:d), kg & $1: 0,25$ & $1: 0,50$ & $1: 0,75$ & $1: 0,41$ \\
\hline
\end{tabular}

De los factores evaluados, resultaron significativos $(\mathrm{p}<0,05)$ para la optimización del rendimiento de la pulpa de ungurahui, la temperatura de maduración $\left(58^{\circ} \mathrm{C}\right)$ y el ratio de pulpeado $(1: 0,41)$. Al respecto6, indica que se realiza en frutas pulposas para ablandar la fruta y facilitar el pulpeado. Por lo expuesto, la temperatura y el tiempo de pre cocción juegan un papel importante en el rendimiento de pulpa, ya que facilita la separación de las demás partes de la fruta, al ablandar sus tejidos. El ungurahui, por sus características sui géneris, requiere de un tratamiento de maduración y posterior pulpeado con adición de agua, para separar óptimamente la pulpa.

En las figuras 4 y 5, se presentan las gráficas de superficie de respuesta y las curvas de contorno, respectivamente, que mejor describen el comportamiento del rendimiento de la pulpa de ungurahui ante la variación de los factores independientes considerados.

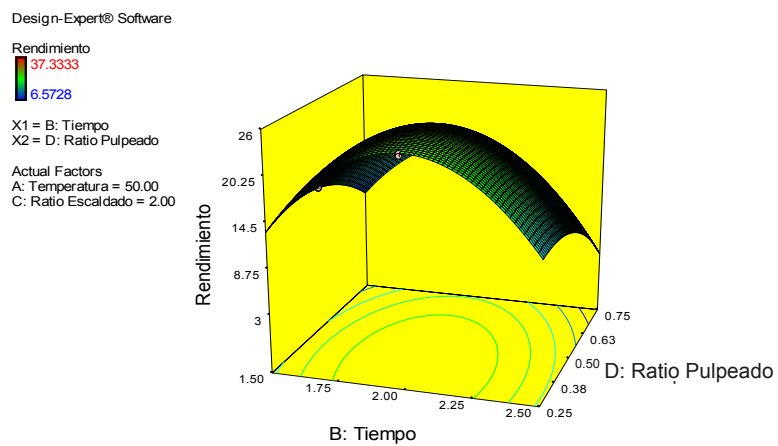

Figura 4. Superficie de respuesta - mayor rendimiento 


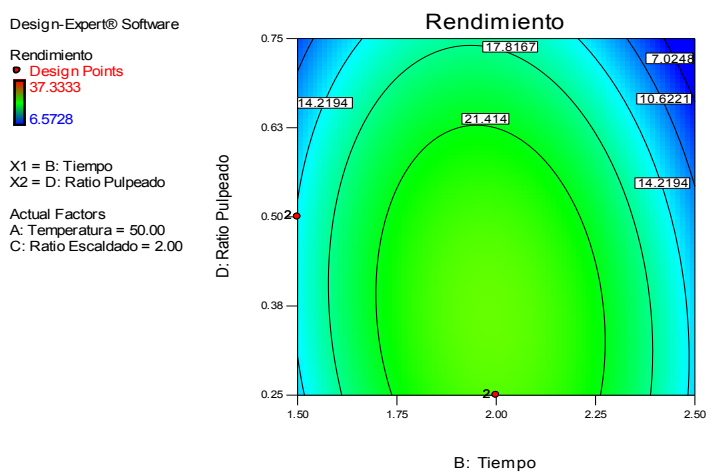

Figura 5. Curvas de contorno de la superficie de respuesta - mayor rendimiento

El análisis de los datos se realizó bajo el criterio de "más es mejor", representado por la forma de montaña de la figura 4 y las crestas ascendentes de la figura 5. En las gráficas de superficie de respuesta, una superficie máxima se representa en forma de montaña, mientras que en las curvas de contorno un máximo se representa por crestas ascendentes ${ }^{10}$.

En la tabla 4 se presentan los valores óptimos de los factores independientes considerados en el análisis del rendimiento de la pulpa de ungurahui, cuya combinación permitió obtener el máximo rendimiento $(37,33 \%)$, de acuerdo al grado de deseabilidad $(0,871)$ establecido en el análisis de superficie de respuesta. El rendimiento es característico de cada materia prima, generalmente en frutas de origen tropical y subtropical es menor que las procedentes de clima templado.

Tabla 4. Niveles óptimos obtenidos para los factores independientes y la variable respuesta (rendimiento), mediante superficie de respuesta y deseabilidad general

\begin{tabular}{|c|c|c|c|c|c|}
\hline & \multicolumn{4}{|c|}{ Factores Independientes } & \multirow[b]{2}{*}{$\begin{array}{c}\text { Rendimiento } \\
\text { (\%) }\end{array}$} \\
\hline & $\begin{array}{c}\text { Temperatura } \\
\left({ }^{\circ} \mathrm{C}\right)\end{array}$ & Tiempo (h) & $\begin{array}{c}\text { Ratio } \\
\text { Maduración (a: b) }\end{array}$ & $\begin{array}{c}\text { Ratio } \\
\text { Pulpeado (c: } \\
\text { d) }\end{array}$ & \\
\hline $\begin{array}{c}\text { Nivel } \\
\text { Óptimo }\end{array}$ & 51,99 & 1,74 & $1: 3$ & 1: 0,75 & 37,33 \\
\hline
\end{tabular}

\section{Determinación de los factores $(p<0,05)$ que influyen en la estabilidad del néctar}

Mediante el análisis de varianza (ANVA) se determinó la significancia $(\mathrm{p}<0,0001)$ del modelo cuadrático descrito en la ecuación de la figura 6. La ecuación muestra el comportamiento de la estabilidad del néctar de ungurahui ante cambios en los factores independientes considerados. 
Estabilidad $(\mathrm{ml})=-10,69+14,48 *$ Dilución $+128,99 * X a n t a n-49,46 * \mathrm{CMC}-342,42 *$ Arábiga $+73,29 *$ Lecitina -116,
79*Dilución*Xantan + 0,34*Dilución*CMC - 14,29*Dilución*Arábiga - 30,71*Dilución*Lecitina + 45,88*Xantan*CMC -
1421,47*Xantan*Arábiga -422,03*Xantan*Lecitina +2814,12*CMC*Arábiga -307,44*CMC*Lecitina +354,31*Arábiga*Lecitina
$-0,01 *$ Dilucion $^{2}+2,70 *$ Xantan $^{2}-0,90 * \mathrm{CMC}^{2}+2,50 *$ Arabiga $^{2}-7,40 *$ Lecitina $^{2}$

Figura 6. Modelo cuadrático para optimizar la estabilidad del néctar de ungurahui

En la tabla 5 se muestran los factores independientes y sus niveles, considerados en el análisis de varianza, para la optimización de la estabilidad del néctar de ungurahui. Al respecto, las frutas tienen sólidos y sustancias espesantes naturales como: pectina y gomas, que le proporcionan una consistencia característica; sin embargo, no todas lo tienen en cantidades apropiadas para lograr la viscosidad y contribuir a evitar la separación de fases en el néctar; por tanto, se recomienda el uso de estabilizantes naturales o comerciales ${ }^{11}$.

Tabla 5. Optimización de la variable respuesta estabilidad

\begin{tabular}{lcccc}
\hline \multicolumn{5}{l}{ Respuesta Óptima } \\
\hline \multicolumn{4}{l}{ Objetivo: Estabilidad óptima del néctar de ungurahui } \\
\multicolumn{4}{l}{ Valor Óptimo = $9,9 \times 10^{-9}$} \\
\multicolumn{1}{c}{ Factor } & Menor & Medio & Mayor & Óptimo \\
Dilución (a:b) & 1 & 2 & 3 & 2,780 \\
Goma Xantan (\%) & 0 & 0,025 & 0,050 & 0,040 \\
CMC (\%) & 0 & 0,050 & 0,100 & 0,020 \\
Goma arábiga (\%) & 0 & 0,025 & 0,050 & 0,050 \\
Lecitina (\%) & 0 & 0,300 & 0,600 & 0,350 \\
\hline
\end{tabular}

De los factores evaluados, resultaron significativos $(\mathrm{p}<0,05)$ para la optimización de la estabilidad del néctar de ungurahui, la concentración de goma xantana $(0,04 \%)$ y de CMC $(0,02 \%)$. Al respecto, las gomas carboximetilcelulosa (CMC), guar, xantana, y arábiga, evitan la sedimentación de la pulpa, aportan consistencia (textura) y mejoran las propiedades sensoriales de los néctares de frutas ${ }^{12}$. Por otro lado, estudios refieren la acción del CMC para mantener suspendidas de manera homogénea las partículas, evitando la sedimentación y aumentando la viscosidad del producto ${ }^{13}$, adicionalmente se pueden utilizar otras gomas de grado alimentario.

Se determinó que para elaborar néctar de ungurahui se requiere la adición de sustancias estabilizantes para integrar los ácidos grasos (característicos de la materia prima), con los otros componentes de la formulación, mejorando así el aspecto general de la bebida.

En las figuras 7 y 8, se presentan las gráficas de superficie de respuesta y las curvas de contorno, que mejor describen el comportamiento de la estabilidad del néctar de ungurahui, ante la variación de los factores independientes considerados. El análisis de los datos se realizó bajo el criterio de "nominal es mejor"10. 



Figura 7. Superficie de respuesta- estabilidad óptima
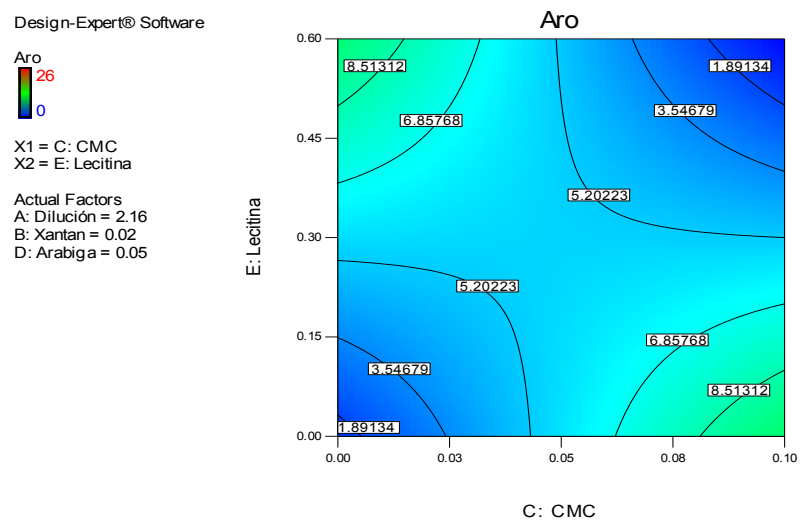

Figura 8. Curvas de nivel de la superficie respuesta - estabilidad óptima

En la tabla 6 se presenta los valores óptimos de los factores independientes considerados en el análisis de la estabilidad del néctar de ungurahui, cuya combinación permitió obtener la mejor estabilidad $2,27 \times 10^{-8} \mathrm{ml}$ (nivel de aro en la superficie de la botella), de acuerdo al grado de deseabilidad $(0,99)$ establecido en el análisis de superficie de respuesta.

Tabla 6. Resumen de los niveles óptimos obtenidos para los factores independientes y la variable respuesta (estabilidad), mediante superficie de respuesta y deseabilidad general

\begin{tabular}{|c|c|c|c|c|c|c|}
\hline & \multicolumn{5}{|c|}{ Factor Independientes } & \multirow{2}{*}{$\begin{array}{r}\text { Estabilidad } \\
\text { Aro (ml) }\end{array}$} \\
\hline & $\begin{array}{c}\text { Dilución } \\
\text { (pulpa, } \\
\text { agua) }\end{array}$ & $\begin{array}{c}\text { Goma } \\
\text { Xantan } \\
(\%)\end{array}$ & $\begin{array}{c}\text { CMC } \\
(\%)\end{array}$ & $\begin{array}{c}\text { Goma } \\
\text { Arábiga } \\
(\%)\end{array}$ & $\begin{array}{c}\text { Lecitina } \\
(\%)\end{array}$ & \\
\hline $\begin{array}{c}\text { Nivel } \\
\text { Óptimo }\end{array}$ & 1,33 & 0,020 & 0,060 & 0,030 & 0,020 & $2,27 \times 10^{-8}$ \\
\hline
\end{tabular}




\section{Análisis sensorial del néctar de ungurahui}

En la tabla 7 se muestra la puntuación total y ponderada del néctar de ungurahui, obtenida en la evaluación sensorial, realizada al producto optimizado.

Tabla 7. Resultados de la evaluación sensorial del néctar de ungurahui optimizado

\begin{tabular}{cccc}
\hline & \multicolumn{3}{c}{ Atributos } \\
\hline \multicolumn{1}{c}{ Análisis } & Sabor & Color & Textura \\
Puntuación total & 604,2 & 574,6 & 640,3 \\
Puntuación ponderada & 6,042 & 5,746 & 6,403 \\
\hline
\end{tabular}

Se obtuvieron valores promedios de aceptabilidad superiores a 5 (de una escala de 10 puntos), en los tres atributos evaluados (sabor, color y textura), siendo el color el de menor puntuación (5 746); dado las características sui géneris de la materia prima que influyen en el néctar. El sabor fue calificado en promedio con 6042 puntos atribuido a las características sensoriales propias del ungurahui y la textura con 6403 puntos debido a la optimización de las gomas utilizadas; que hicieron que el néctar obtenido sea aceptado por los jueces evaluadores.

\section{Análisis microbiológico del néctar de ungurahui}

El producto obtenido mostró estabilidad microbiológica, el número de aerobios mesofilos viables $(10 \mathrm{UFC} / \mathrm{ml})$, levaduras $(<1 \mathrm{UFC} / \mathrm{ml})$ y mohos $(<1 \mathrm{UFC} / \mathrm{ml})$, fueron mínimos, estando por debajo de los límites microbiológicos establecido por el MINSA ${ }^{14}$ y APHA/CMMEF, reflejando las buenas prácticas de manufactura y la calidad de la fruta con la que se trabajó.

\section{CONCLUSIONES}

- Los parámetros de los procesos de maduración y pulpeado, que optimizaron el rendimiento de la pulpa de ungurahui, fueron: temperatura $\left(51,99^{\circ} \mathrm{C}\right)$; tiempo $(1,74 \mathrm{~h})$; ratio fruta: agua de maduración (1:3) y ratio fruta: agua de pulpeado (1:0,75). Bajo estas condiciones se obtuvo $37,33 \%$ de rendimiento.

- Los parámetros del proceso de estandarizado, que optimizaron la estabilidad del néctar de ungurahui, fueron: dilución (1: 1,33); goma xantana $(0,020 \%)$; CMC $(0,060 \%)$; goma arábiga $(0,030 \%)$ y lecitina $(0,020 \%)$, obteniendo $2,27 \times 10^{-8} \mathrm{ml}$ (nivel de aro en la superficie de la botella), como indicativo de estabilidad.

- La evaluación sensorial del producto obtenido indicó una aceptabilidad promedio superior al $50 \%$ tanto para textura, sabor y color, destacando la textura como la de mayor aceptabilidad.

- $\quad$ La composición fisicoquímica del ungurahui y del néctar fue: 313,82 y 57,30; 34,79 y $86,90 \% ; 24,03$ y $11 \% ; 21,30$ y $1,30 \% ; 12,25$ y $0,40 \% ; 6,5$ y $0 \% ; 1,13$ y $0,40 \% 0,051$ 
y $2,11 \% ; 0,37$ y $13 ; 5,49$ y 3,71 ; de cal/100g de fruta, humedad, carbohidratos, grasa, fibra, proteínas, cenizas, acidez, ${ }^{\circ} \mathrm{Brix}$ y $\mathrm{pH}$, respectivamente; adicionalmente el néctar reportó 0,99 de aw.

- Los resultados microbiológicos del néctar de ungurahui se encuentran dentro de lo establecido por la normativa nacional (R.M. N591-2008-MINSA) para este tipo de bebidas, indicando así que fue procesado apropiadamente.

\section{REFERENCIAS BIBLIOGRÁFICAS}

1. Aguirre-Calderón O. Manejo Forestal en el Siglo XXI. 2015. Disponible en: http:// www.scielo.org.mx/scielo.php?script=sci_arttext\&pid=S1405-04712015000400002. pdf.

2. Balslev H, Ríos M, Quezada G,, Nantipa B. Palmas útiles en la Cordillera de los Huacamayos. Colección de Manuales de Aprovechamiento Sustentable de Bosque. Probona-Quito. 1997; 1: 1-57.

3. Villachica H. Frutales y Hortalizas Promisorios de la Amazonia. Tratado de Cooperación Amazónica, Secretaria Pro - Tempore. Lima - Perú. 1996; 367.

4. AOAC International. Official Methods of Analysis of AOAC International. Rockville Maryland USA. 2016. ISBN: 0-935584-87-0.

5. Collazos C., Phlip W., Viñas E., Alvistur J., Urquieta A., Vásquez J. La composición de alimentos de mayor consumo en el Perú. 6ta Edición. Ministerio de Salud- Instituto Nacional de Nutrición. Lima- Perú. 1993; 60.

6. Guevara A. Elaboración de pulpas, zumos, néctares, deshidratados, osmodeshidratados y fruta confitada. Universidad Nacional Agraria La Molina - Facultad de Industrias Alimentarias - Departamento de Tecnología de Alimentos y Productos Agropecuarios. Lima - Perú. 2015; 5.

7. McLaren K. Food colorimetry- Developments in food colours I. Walford J, editor. London, U.K. 1996; 9: 27-45.

8. APHA/CMMEF. Compendium of Methods for the Microbiological Examination of Foods. 4th Edition. 2001; Chapter 7: 64-65.

9. Márquez CC, Otero EC, Cortes RM. Cambios fisiológicos, texturales, fisicoquímicos y micro estructurales del tomate de árbol (Chyphomandra betacea S.) en poscosecha. Compendiado en: VITAE, Revista de la Facultad de Química Farmacéutica de la Universidad de Antioquia. Vol. 14, No. 2. Colombia. 2007; 10-16.

10. Gutiérrez H., De La Vara R. Análisis y diseño de experimentos. Primera edición. Editorial Mc Graw Gill Interamericana Editores S.A. México. 2008; 468.

11. Coronado M., Hilario R. Procesamiento de alimentos para pequeñas y microempresas agroindustriales. Unión Europea, CIED, EDAD, CEPCO. Lima- Perú. 2006, 120.

12. Pastor MV, Costell E, Duncan L. Efeccts of hydrocolloids and aspartame on sensory viscosity and sweetener of low calorie peach nectars. Journal Texture Studied. 1996; 27 : 61-79. 
13. Vargas R. y Pisfil E. Estudio químico bromatológico y elaboración de néctar de Mespilus germánica L. (níspero de palo) procedente de la provincia de Vilcashuamán, departamento de Ayacucho. Lima-Perú. 2008; 80.

14. MINSA. Norma sanitaria que establece los criterios microbiológicos de calidad e inocuidad para los alimentos y bebidas de consumo humano. El Peruano, 27 de Agosto del 2008. Disponible en: www.sanipes.gob.pe/archivos/biblioteca/N_14_RM_591_2008_ MINSA.pdf 